\title{
Meloxicam suppresses hepatocellular carcinoma cell proliferation and migration by targeting COX-2/PGE2-regulated activation of the $\beta$-catenin signaling pathway
}

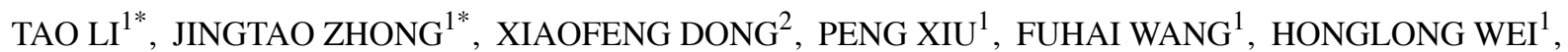 \\ XIN WANG ${ }^{1}$, ZONGZHEN XU $^{1}$, FENG LIU ${ }^{1}$, XUEYING SUN ${ }^{3,4}$ and JIE LI $^{1}$ \\ ${ }^{1}$ Department of General Surgery, Qianfoshan Hospital, Shandong University, Jinan, Shandong 250014; \\ ${ }^{2}$ Department of Hepatobiliary Surgery, The People's Hospital of Guangxi Zhuang Autonomous Region, Nanning, \\ Guangxi 530021; ${ }^{3}$ The Hepatosplenic Surgery Center, Department of General Surgery, The First Affiliated Hospital \\ of Harbin Medical University, Harbin, Heilongjiang 150081, P.R. China; ${ }^{4}$ Department of Molecular Medicine and \\ Pathology, Faculty of Medical and Health Sciences, University of Auckland, Auckland 1142, New Zealand
}

Received January 11, 2016; Accepted February 17, 2016

DOI: $10.3892 /$ or.2016.4764

\begin{abstract}
Recurrence and metastasis are the two leading causes of poor prognosis of hepatocellular carcinoma (HCC) patients. Cyclooxygenase (COX)-2 is overexpressed in many types of cancers including $\mathrm{HCC}$ and promotes its metastasis. Meloxicam is a selective COX-2 inhibitor that has been reported to exert an anti-proliferation and invasion/migration response in various tumors. In this study, we examined the role of meloxicam on HCC cell proliferation and migration and explored the molecular mechanisms underlying this effect. We found that meloxicam inhibited HCC cell proliferation and had a cell cycle arrest effect in human HCC cells. Furthermore, meloxicam suppressed the ability of HCC cells expressing higher levels of COX-2 and prostaglandin E2 (PGE2) to migration via potentiating expression of E-cadherin and alleviating expression of matrix metalloproteinase (MMP)-2 and -9 . COX-2/PGE2 has been considered to activate the $\beta$-catenin signaling pathway which promotes cancer cell migration. We found that treatment with PGE2 significantly enhanced nuclear accumulation of $\beta$-catenin and the activation of GSK $3 \beta$ which could be reversed by meloxicam in HCC cells. We also observed that HCC cell migration and upregulation of the level of MMP-2/9 and downregulation of E-cadherin induced by PGE2 were suppressed by FH535, an inhibitor of $\beta$-catenin. Taken together, these findings provide a new treatment strategy against HCC proliferation and migration.
\end{abstract}

Correspondence to: Dr Jie Li, Department of General Surgery, Qianfoshan Hospital, Shandong University, 16766 Jingshi Road, Jinan, Shandong 250014, P.R. China

E-mail: 1ijieqfs@126.com

${ }^{*}$ Contributed equally

Key words: hepatocellular carcinoma, meloxicam, migration, COX-2/PGE2, $\beta$-catenin signaling pathway

\section{Introduction}

Hepatocellular carcinoma (HCC) ranks as the sixth most common malignancy as well as the third most common cause of cancer leading to death worldwide, and its incidence continues to rise $(1,2)$. Hepatitis virus infection (hepatitis $B$ or $\mathrm{C}$ viruses), alcohol-related liver cirrhosis, and nonalcoholic steatohepatitis have been recognized as the main risk factors for HCC (3). Despite improvements in surgical and medical treatments in the past decade, the outcome of patients with HCC remains unsatisfactory. Recurrence and metastasis are the two leading causes of poor prognosis of HCC patients (4). Therefore, there is an urgent need to understand the cellular mediators that contribute to the invasion and migration of HCC and explore new therapeutic strategies targeting these mediators.

Cyclooxygenase-2 (COX-2) has been considered a potential mediator of invasion and migration in a number of malignant diseases (5-8). It has also been reported to be associated with poor prognosis in many cancers $(9,10)$. Overexpression of COX-2 enhances the extent of prostaglandin E2 (PGE2) which is the main metabolite of COX-2 and a ligand of $\mathrm{G}$ proteincoupled receptors including EP1, EP2, EP3, and EP4. Recently, several studies reported that COX-2/PGE2 stimulates AKT, $\mathrm{NF}-\kappa \mathrm{B}$, and ERK1/2 signaling pathways to promote tumor angiogenesis, apoptosis, and invasiveness (8,11-13). Our previous studies demonstrated that meloxicam, a selective COX-2 inhibitor, suppresses HCC cell proliferation, migration, and invasion via regulating expression of matrix metalloproteinase (MMP)-2 and E-cadherin in a COX-2-dependent manner (14). However, the precise mechanisms of migration and invasion of HCC are largely unknown.

Accumulating evidence has demonstrated that COX-2/ PGE2 is associated with the $\beta$-catenin signaling pathway contributing to the growth of many cancers, such as skin (15), breast (16), neuroblastoma (17) and colorectal cancer (18). $\beta$-catenin is a $90 \mathrm{kD}$ cytosolic protein and an important component of the Wnt signaling pathway. In the absence model of 
Wnt signaling, $\beta$-catenin is recruited to the phosphorylation/ destruction complex. Disturbance of the complex promotes the phosphorylation of $\beta$-catenin by glycogen synthase kinase- $3 \beta$ (GSK-3 $\beta)$ and casein kinase $1 \alpha(\mathrm{CK} 1 \alpha)$ leading to the proteasomal degradation of $\beta$-catenin (19). $\beta$-catenin accumulation eventually leads to its nuclear translocation and then it binds to members of the TCF/LEF family of transcription factors, thus regulating expression of various target genes which are associated with many cellular processes including cell survival, proliferation, and migration (20-22).

Meloxicam (Mel) is an NSAID that specifically inhibits COX-2. This selective COX-2 inhibitor has been demonstrated to exert an anti-invasion response in various tumors $(5,23,24)$ including $\mathrm{HCC}$ cancer $(14,25,26)$. However, whether meloxicam inhibits HCC cell invasion/migration by targeting COX-2/PGE2-regulated activation of the $\beta$-catenin signaling pathway remains unclear. In this study, we investigated the effects of meloxicam on the proliferation and migration potential of HCC cells and explored whether the antitumor effect of meloxicam is associated with the inactivation of the $\beta$-catenin signaling pathway and whether COX-2/PGE2 plays any part in this process.

\section{Materials and methods}

Cell culture. Of the 5 human HCC cell lines, HepG2, Bel-7402, and Huh-7 were obtained from the American Type Culture Collection (ATCC, Rockville, MD, USA), and SMMC-7721 and SMMC-7402 were obtained from the Type Culture Collection Cell Bank, Chinese Academy of Science (Shanghai, China). The cells were routinely cultured in RPMI-1640 medium (Gibco)/DMEM (Hyclone) supplemented with 10\% fetal bovine serum (Gibco) and $1 \%$ antibiotics at $37^{\circ} \mathrm{C}$ in $95 \%$ air and $5 \% \mathrm{CO}_{2}$.

Reagents and antibodies. Meloxicam was obtained from Merck Millipore (Darmstadt, Germany). PGE2 and FH535 were obtained from Sigma-Aldrich (San Diego, CA, USA). Primary antibodies to COX-2, p21, p27, MMP-2, and MMP-9 were obtained from Cell Signaling Technologies (Danvers, MA, USA). Antibodies to E-cadherin, $\beta$-catenin, GSK-3 $\beta$, p-GSK-3 $\beta$, Histone H3, and GAPDH were obtained from Abcam (Cambridge, UK).

Cell viability analysis. The effect of meloxicam on the cell viability of HCC cells was determined using the Cell Counting Kit-8 (CCK-8, Dojindo Molecular Technologies, Inc., Kumamoto, Japan) assay as previously described (25).

Enzyme-linked immunosorbent assay (ELISA). The concentrations of PGE2 in supernatants of cell cultures were measured using the PGE2 ELISA Assay kit (R\&D Systems, Inc., Minneapolis, MN, USA) according to the manufacturer's instructions.

Cell cycle analysis. HepG2 and SMMC-7721 cells were grown in medium as mentioned above. At $50 \%$ confluency, cells were treated with meloxicam or not for $24 \mathrm{~h}$. Cells were collected and processed for cell cycle analysis. Briefly, $5 \times 10^{4}$ cells were suspended in $0.5 \mathrm{ml}$ of PI solution, and incubated for $30 \mathrm{~min}$ in the dark according to the manufacturer's instruction. The cell cycle distribution was analyzed by FACS flow cytometry.

Cell migration analysis. The methods were previously described $(14,25)$. In brief, $1 \times 10^{5}$ cells in $300 \mu 1$ of RPMI-1640 medium/DMEM (with 1\% FBS) containing meloxicam or PGE2 alone or in combination were seeded into the upper chamber of a Transwell chamber (Corning, New York, USA). The bottom wells of the chambers were filled with $500 \mu \mathrm{l}$ RPMI-1640 medium/DMEM containing 10\% fetal bovine serum. After $48 \mathrm{~h}$ incubation, the chambers were fixed with $95 \%$ ethanol and then stained with $1 \%$ crystal violet. Images of three different fields (x100 magnification) were captured from each membrane, and the number of migrated cells counted.

Total RNA extraction and real-time PCR. Total RNA was extracted from the cells using TRIzol reagent (Invitrogen, Carlsbad, CA, USA), and cDNA was synthesized by using a cDNA synthesis kit (Invitrogen). The reaction mixtures for quantitative RT-PCR were prepared as previously described (14). The primers targeting MMP-2 were (5'-TGA CGGTAAGGACGGACTC-3'; 5'-ATACTTCACACGGAC CACTTG-3'), MMP9 (5'-CCTCTGGAGGTTCGACGTGA-3'; 5'-TAGGCTTTCTCTCGGTACTGGAA-3'), E-cadherin (5'-TGCCCAGAAAATGAAAAAGG-3'; 5'-GGATGACAG CGTGAGAGA-3'), and GAPDH (5'-TTACTCCTTGGAGGC CATGTGGGC-3'; 5'-ACTGCCACCCAGAAGACTGTGGA TGG-3'). Expression levels were normalized to GAPDH. All protocols were carried out according to the manufacturer's instructions. Real-time PCR was performed using MX3000P Real-time PCR systems (Stratagene, Wilmington, DE, USA). Experiments were performed in triplicate, and the data were calculated by $\Delta \Delta C t$ methods.

Western blot analysis. The method was previously described (27). After different treatments, protein concentrations in cell extracts were determined (Bio-Rad, Richmond, CA, USA). Equal amounts of each sample were resolved in SDS-PAGE gels, then transferred to a polyvinylidene fluoride (PVDF) membrane (Millipore, Billerica, MA, USA), and probed with the primary antibodies described in reagents and antibodies.

siRNA transfection. $\beta$-catenin and COX-2 siRNA were purchased from Santa Cruz Biotechnology, Inc. (Santa Cruz, CA, USA) and transfection was performed using Lipofectamine 2000 Transfection Reagent (Invitrogen) according to the manufacturer's instructions.

Statistical analysis. Data are presented as the mean \pm standard deviation (SD) and analyzed by one-way ANOVA followed by Dunnett's test with SPSS software (version 17.0, SPSS China, Shanghai, China), with values of $\mathrm{P}<0.05$ considered statically significant.

\section{Results}

Effects of meloxicam on the PGE2 level and the proliferation potential of $\mathrm{HCC}$ cells in vitro. First, we examined expression of COX-2 protein in HCC cells by western blot analysis. As shown in Fig. 1A, HCC cell lines exhibited different levels 
A

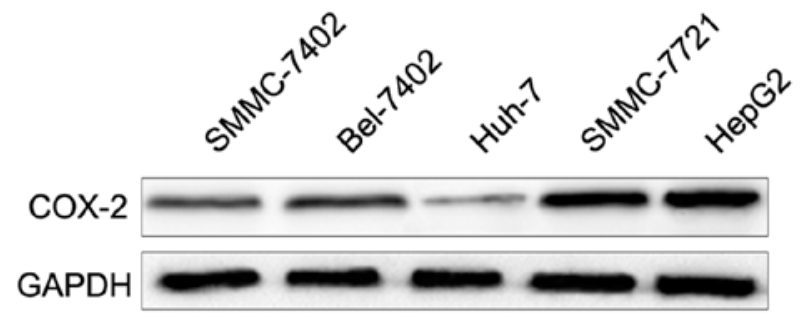

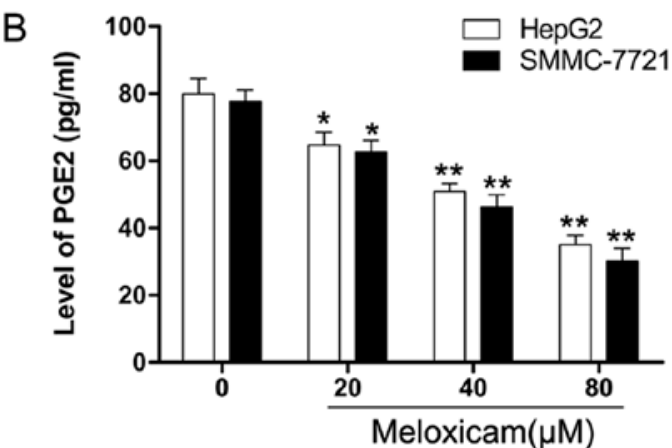

C

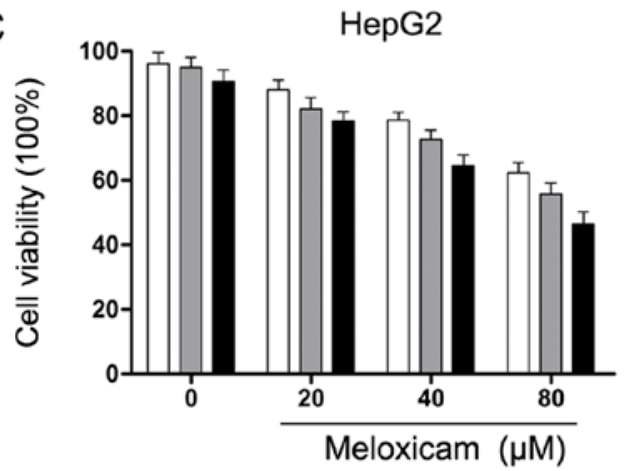

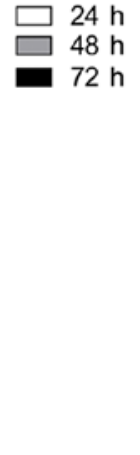

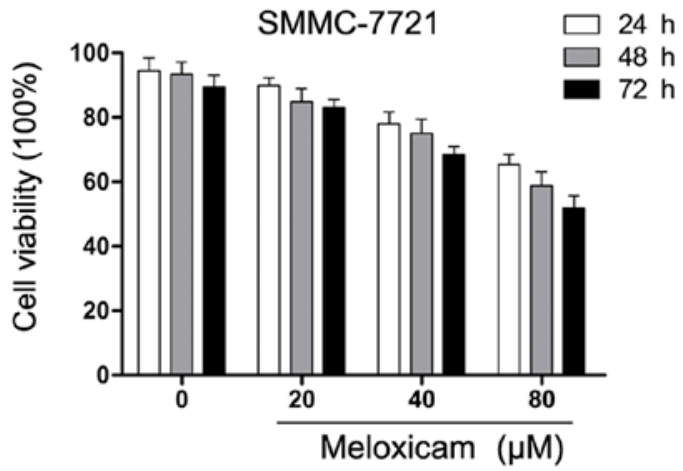

Figure 1. Effects of meloxicam on the PGE2 level and the proliferation potential of HCC cells in vitro. (A) Expression of COX-2 in HCC cell lines SMMC-7402, Bel-7402, Huh-7, SMMC-7721, and HepG2 was detected by western blotting. GAPDH was measured as the loading control. Results shown are representative of at least three independent experiments. (B) PGE2 levels of HCC cells were determined by ELISA following treatment with various concentration of meloxicam $(0-80 \mu \mathrm{M})$. Data are presented as means \pm SD of three independents experiments. ${ }^{*} \mathrm{P}<0.05,{ }^{* * *} \mathrm{P}<0.01$ vs. control. (C) Cell vitality was assessed by the $\mathrm{CCK}-8$ assay. HepG2 and SMMC-7721 cells were exposed to various concentrations of meloxicam $(0-80 \mu \mathrm{M})$ for 24,48 , or $72 \mathrm{~h}$. Data are expressed as the percentage of control cells and are the means \pm SD of three separate experiments.

A
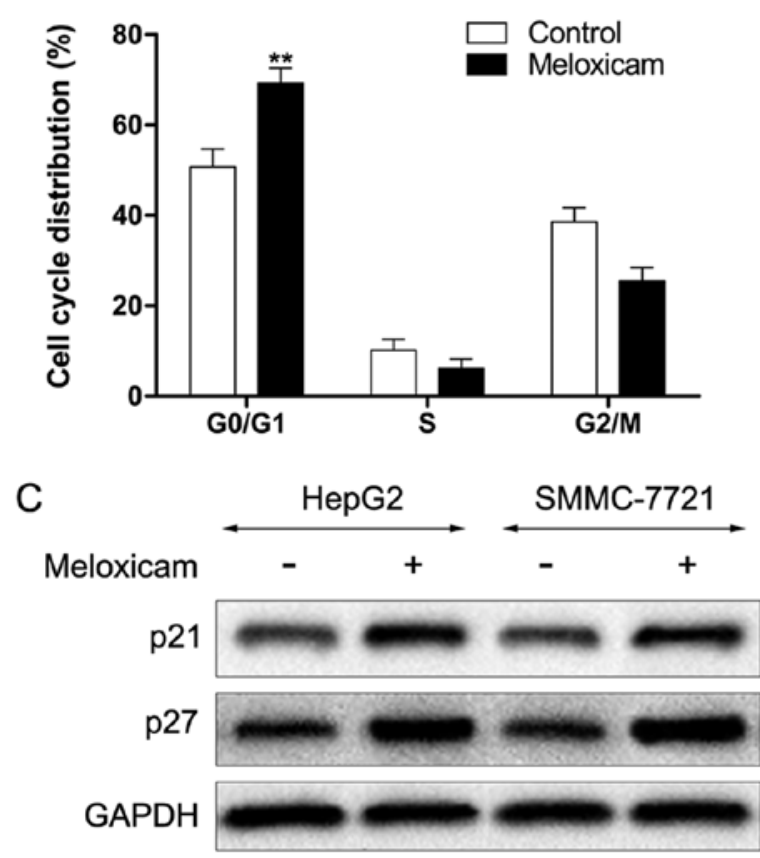

B

SMMC-7721
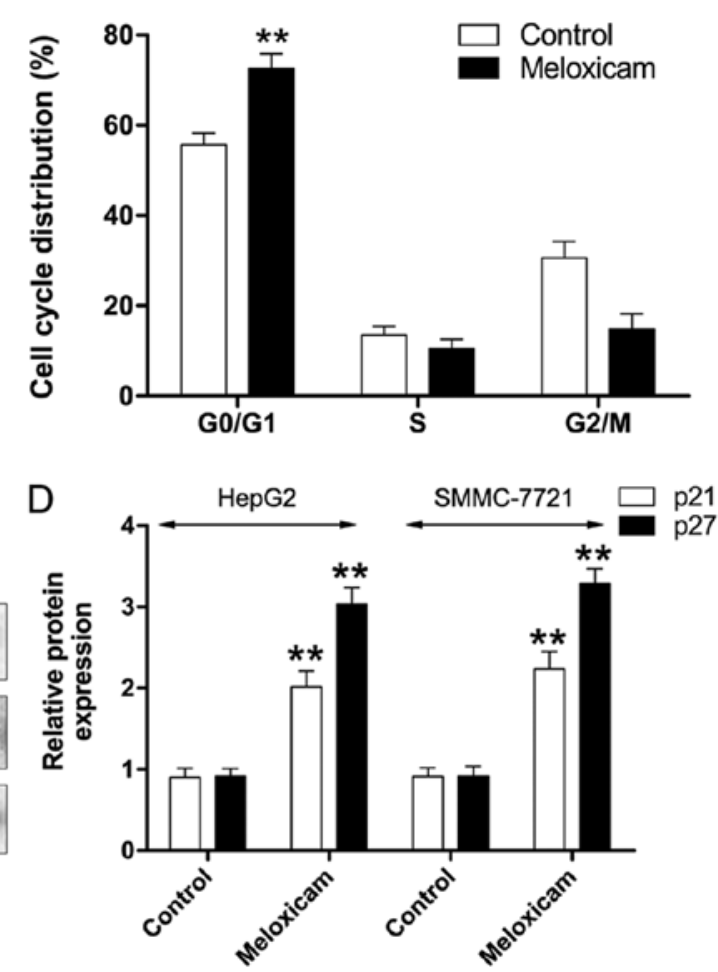

Figure 2. Meloxicam induces cell cycle arrest at the G1 phase in HepG2 and SMMC-7721 cells in vitro. (A and B) HepG2 and SMMC-7721 cells were treated with meloxicam $(80 \mu \mathrm{M})$ or control for $24 \mathrm{~h}$ and then cell cycle analysis was measured as described in Materials and methods. Data are presented as means \pm SD of three independents experiments. ${ }^{* *} \mathrm{P}<0.01 \mathrm{vs}$. control. (C and D) Expression levels of p21 and p27 at $24 \mathrm{~h}$ after meloxicam treatment were analyzed by western blotting. GAPDH was measured as the loading control. Results shown are representative of at least three independent experiments. ${ }^{* *} \mathrm{P}<0.01 \mathrm{vs.}$ control. 
A
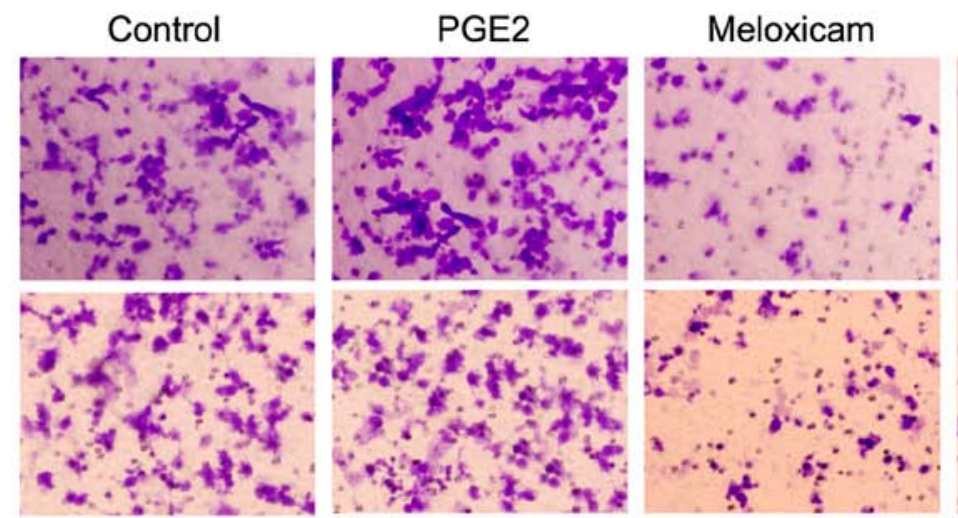

Mel+PGE2

B

HepG2

SMMC-7721
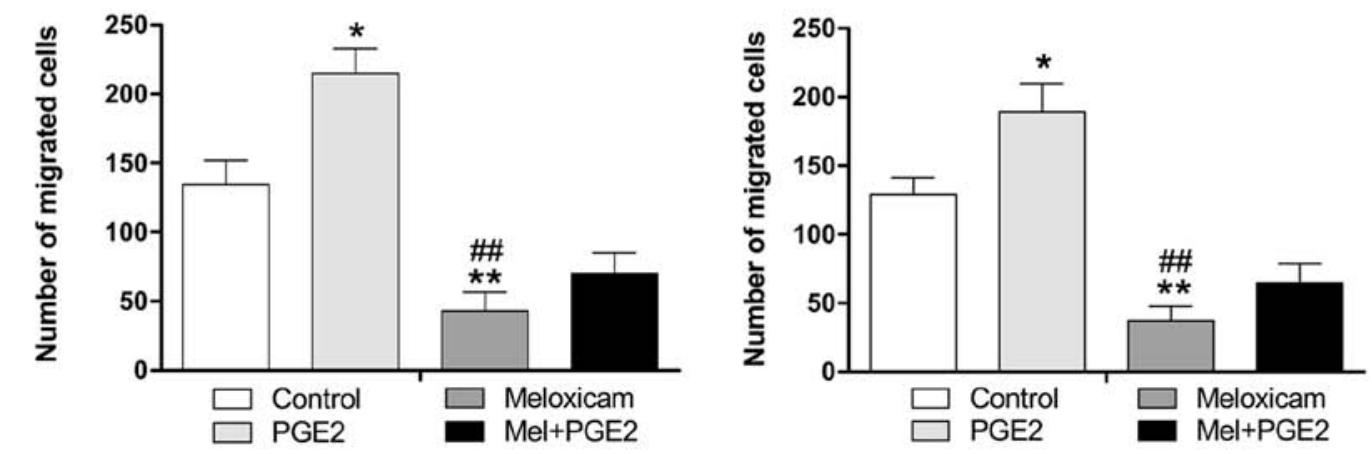

C


Figure 3. Effects of meloxicam on PGE2-induced HCC cell migration and endogenous expression of COX-2. (A and B) Representative images were taken from HepG2 and SMMC-7721 cells incubated with PGE2 $(10 \mu \mathrm{M})$, meloxicam $(80 \mu \mathrm{M})$, or a combination and subjected to cell migration assays as described in Materials and methods (magnification, $\mathrm{x} 100$ ). ${ }^{*} \mathrm{P}<0.05,{ }^{* * *} \mathrm{P}<0.01$ vs. control, ${ }^{\# \#} \mathrm{P}<0.01$ vs. PGE2. (C) Cells were treated with various concentrations of meloxicam for $24 \mathrm{~h}$ and then harvested and cell lysates were subjected to western blot analysis for the measurement of COX-2 levels. GAPDH was measured as the loading control. Results shown are representative of at least three independent experiments. ${ }^{* *} \mathrm{P}<0.01 \mathrm{vs}$. control, ${ }^{\sharp} \mathrm{P}<0.05 \mathrm{vs}$. meloxicam ( $\left.40 \mu \mathrm{M}\right)$ group. (D) Transfection of HepG2 and SMMC-7721 cells with COX-2 siRNA significantly decreased cell migration. ${ }^{* *} \mathrm{P}<0.01$ vs. control.

of COX-2 and the results were consistent with our previous data (14). Based on the data, HepG2 and SMMC-7721 cells were chosen for the following experiments. Next, the PGE2 level of HepG2 and SMMC-7721 cells was determined by ELISA analysis. As shown in Fig. 1B, the level of PGE2 was significantly decreased with meloxicam treatment in a dose-dependent manner. HCC cells were exposed to various concentrations of meloxicam $(0-80 \mu \mathrm{M})$ for 24,48 , or $72 \mathrm{~h}$ and cell viability was determined using the $\mathrm{CCK}-8$ assay. As shown in Fig. 1C, the viability of HCC cells exposed to meloxicam was significantly reduced in a time- and concentration-dependent manner. This result showed the efficacy of meloxicam against HCC cell proliferation.

Effects of meloxicam on the cell cycle of HCC cells in vitro. In our previous study, we demonstrated that meloxicam has a cell cycle arrest effect in human HCC cells (25). In the current work, we further investigated the mechanism of meloxicam in regulating the cell cycle in HepG2 and SMMC-7721 cells. As shown in Fig. 2A and B, with meloxicam treatment both types of HCC cells were suppressed in the G1 phase after $24 \mathrm{~h}$ treatment. Furthermore, we found that expression levels of cyclin-dependent kinase (CDK) inhibitor proteins p21 and p27 in HepG2 and SMMC-7721 cells were significantly enhanced after treatment with meloxicam (Fig. 2C and D).

Effects of PGE2 and meloxicam on HCC cell migration in vitro. Previous studies reported that COX-2/PGE2 plays an important role in exerting pro-invasion/migration effects in many cancers (28-30). Here, we investigated the antimigration effects of meloxicam on HCC cells with or without treatment with exogenous PGE2. As shown in Fig. 3A and B, 
A


SMMC-7721


Figure 4. The effects of meloxicam on protein and mRNA expression of MMP-2, MMP-9, and E-cadherin in HCC cells in vitro. (A and B) HepG2 and SMMC-7721 cells were treated with PGE2 $(10 \mu \mathrm{M})$, meloxicam $(80 \mu \mathrm{M})$, or a combination for $24 \mathrm{~h}$ and then total protein was extracted. Western blotting was performed to detect protein levels of MMP-2, MMP-9, and E-cadherin. GAPDH was measured as the loading control. Results shown are representative of at least three independent experiments. ${ }^{* *} \mathrm{P}<0.01$ vs. control. (C) Cells were treated with PGE2 or meloxicam and total RNA was extracted. mRNA expression of MMP-2, MMP-9, and E-cadherin was determined by real-time quantitative RT-PCR. GAPDH served as an internal control. Data represent three independent experiments. ${ }^{* *} \mathrm{P}<0.01$ vs. control.

treatment with PGE2 significantly enhanced the migration of HepG2 and SMMC-7721 cells. However, this effect could be reversed by meloxicam. Moreover, we examined expression of COX-2 in HepG2 and SMMC-7721 cells treated with meloxicam or not using western blot analysis. As expected, we found that treatment of HepG2 and SMMC-7721 cells with meloxicam for $24 \mathrm{~h}$ induced a marked reduction of COX-2 expression in these cells (Fig. 3C). The effect of COX-2/PGE2 in HCC cell migration was further verified by downregulation of COX-2 by siRNA. As shown in Fig. 3D, transfection with COX-2 siRNA notably decreased the migration in HepG2 and SMMC-7721 cells. These results revealed that the suppression of endogenous levels of COX-2/ PGE2 expression is associated with the inhibition of HCC cell migration.
Effects of PGE2 and meloxicam on MMP-2/9 and E-cadherin expression in $\mathrm{HCC}$ cells. Since the downregulation of E-cadherin and upregulation of expression of MMP-2/9 is associated with enhancement in migration/invasion of cancer cells (31-34), we investigated protein expression and mRNA of MMP-2/9 and E-cadherin in HepG2 and SMMC-7721 cells. As shown in Fig. 4A and B, the level of MMP-2 and MMP-9 was significantly enhanced by PGE2 and reversed by meloxicam. Expression of E-cadherin was decreased by treatment with PGE2 whereas it was increased after being exposed to meloxicam. The results of RT-PCR showed similar effects in mRNA expression of MMP-2/9 and E-cadherin (Fig. 4C). These results suggested that meloxicam decreases MMP-2/9 activity and enhances the level of E-cadherin to inhibit the migration of HCC cells. 
A

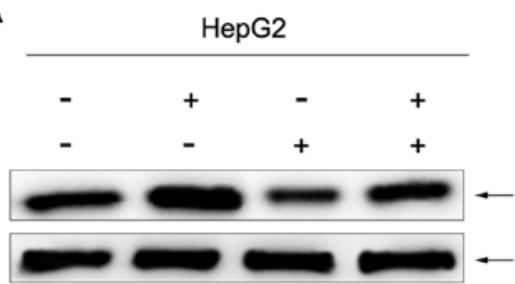

B
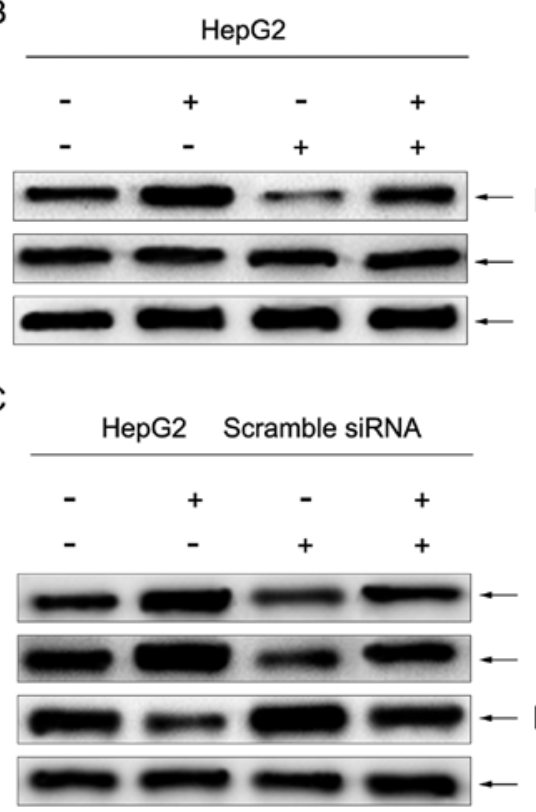
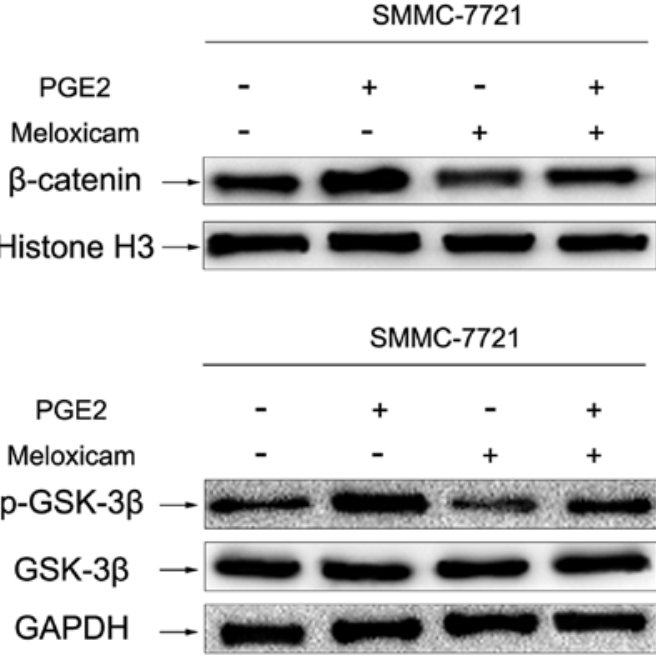

GAPDH

D

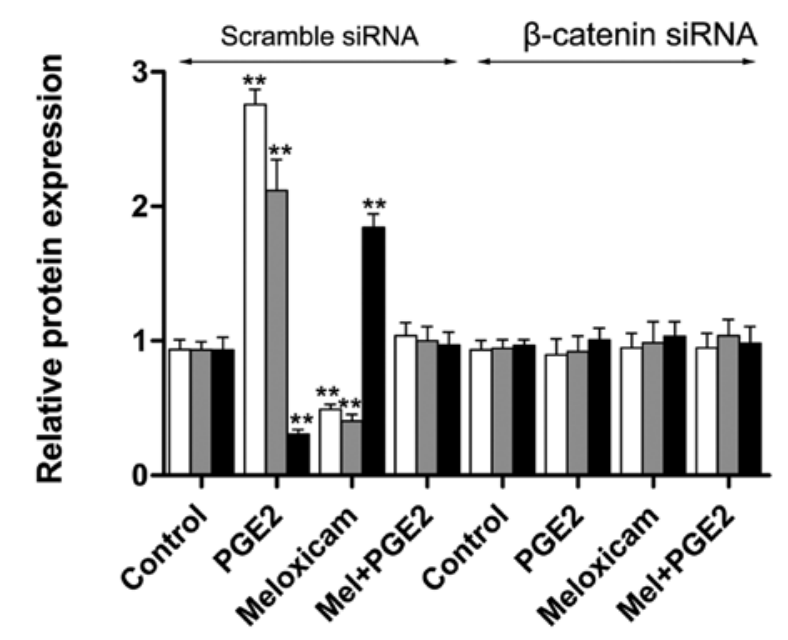

PGE2

Meloxicam



MMP-2

MMP-9

$\rightarrow$

$\begin{array}{llll}- & + & - & + \\ - & - & + & +\end{array}$

E-cadherin $\rightarrow$

GAPDH
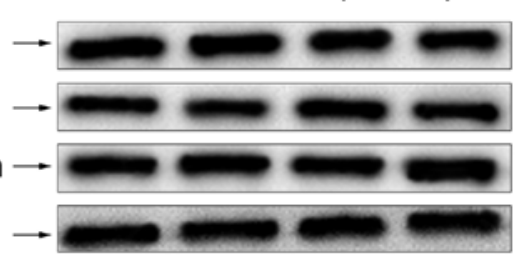

D

Figure 5. The effects of meloxicam on the $\beta$-catenin signaling pathway in HCC cells in vitro. (A) The effect of meloxicam ( $80 \mu \mathrm{M})$ on $\beta$-catenin expression in the nucleus of HepG2 and SMMC-7721 cells with or without PGE2 $(10 \mu \mathrm{M})$. Histone H3 was measured as the loading control. (B) The effect of meloxicam $(80 \mu \mathrm{M})$ on the GSK3 $\beta$ phosphorylation level in HCC cells with or without PGE2 $(10 \mu \mathrm{M})$. GAPDH was measured as the loading control. (C and D) HepG2 cells were transfected with scramble or $\beta$-catenin siRNA and treated with PGE2 $(10 \mu \mathrm{M})$ or meloxicam $(80 \mu \mathrm{M})$ and total protein was extracted. Western blotting was performed to detect protein levels of MMP-2, MMP-9, and E-cadherin. GAPDH was measured as the loading control. Data represent three independent experiments. ${ }^{* *} \mathrm{P}<0.01$ vs. control.

Effects of PGE2 and meloxicam on the $\beta$-catenin signaling pathway in HCC cells in vitro. Accumulating evidence has demonstrated that PGE2 exerts a crucial role in promoting migration and regulating expression of MMP-2/9 and E-cadherin via the $\beta$-catenin signaling pathway $(15,35)$. In this study, we investigated the role of meloxicam on the $\beta$-catenin signaling pathway. As depicted in Fig. 5A, treatment with PGE2 significantly enhanced nuclear accumulation of $\beta$-catenin which could be reversed by meloxicam in HepG2 and SMMC-7721 cells. Several studies reported that PGE2 can inactivate GSK $3 \beta$ and result in a consequent intracellular accumulation of $\beta$-catenin (36). Thus, we investigated the role of meloxicam on the level and activation of GSK3 $\beta$. We found that treatment with meloxicam significantly inhibited the phosphorylation of GSK3 $\beta$, however, expression of GSK3 $\beta$ was only slightly changed (Fig. 5B). Furthermore, knockdown of $\beta$-catenin by siRNA was utilized to examine expression of MMP-2/9 and E-cadherin. As shown in Fig. 5C and D, downregulation of 
A

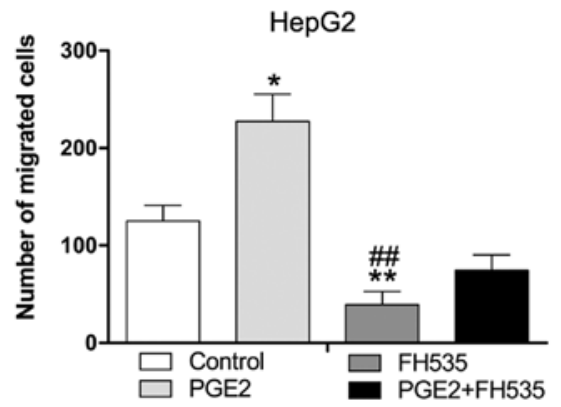

B

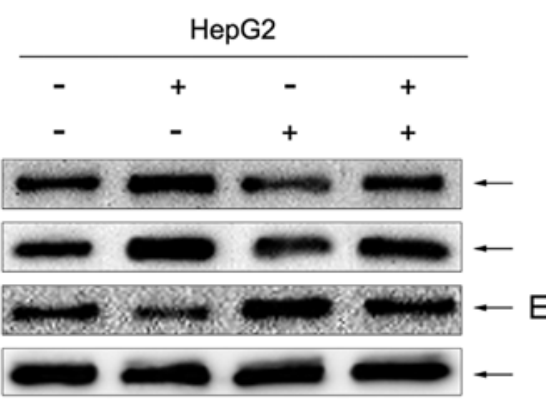



PGE2

FH535

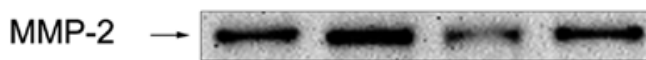

MMP-9 $\rightarrow$

-cadherin $\rightarrow$

GAPDH $\rightarrow$
C

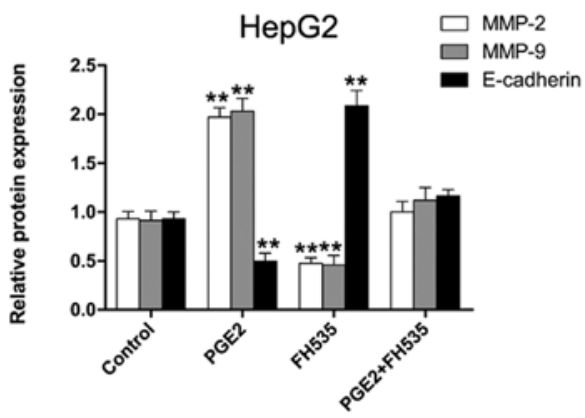

D

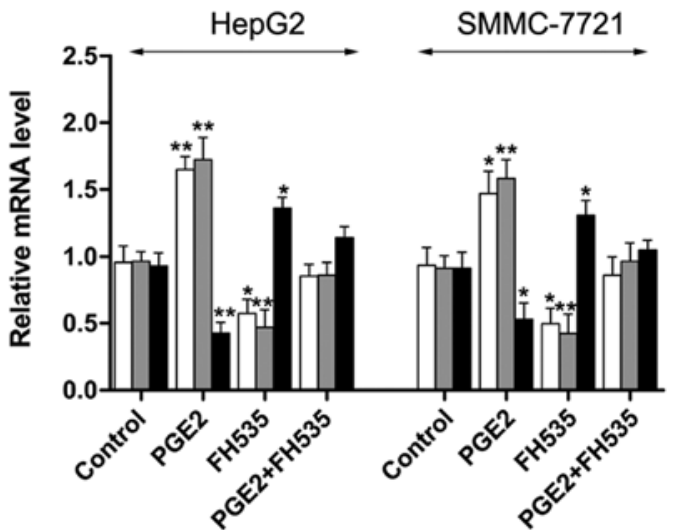

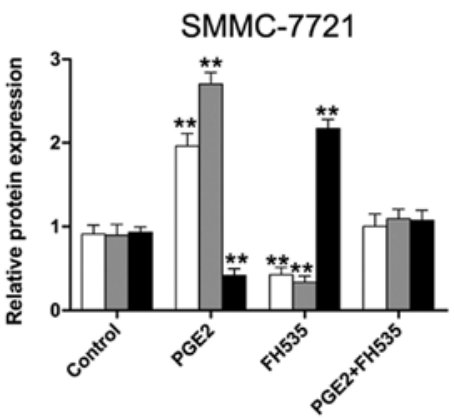

$\square$ MMP-2

- E-cadherin

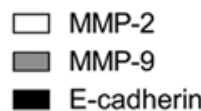

E-cadherin

igure 6. FH535 inhibits PGE2-enhanced cell migration of HCC cells in vitro. (A) FH535 suppresses PGE2-enhanced migration of HepG2 and SMMC-772 cells. ${ }^{*} \mathrm{P}<0.05,{ }^{* *} \mathrm{P}<0.01$ vs. control, ${ }^{\# /} \mathrm{P}<0.01$ vs. PGE2. (B and C) HCC cells were treated with PGE2 $(10 \mu \mathrm{M}), \mathrm{FH} 535(5 \mu \mathrm{M})$, or a combination and total protein was extracted. Western blotting was performed to detect protein levels of MMP-2, MMP-9, and E-cadherin. GAPDH was measured as the loading control. Data represent three independent experiments. ${ }^{* *} \mathrm{P}<0.01$ vs. control. (D) HCC cells were treated with PGE2 $(10 \mu \mathrm{M})$, FH535 $(5 \mu \mathrm{M})$, or a combination and total RNA was extracted. mRNA expression of MMP-2, MMP-9, and E-cadherin was determined by RT-PCR. GAPDH served as an internal control. Data represent three independent experiments. ${ }^{*} \mathrm{P}<0.05,{ }^{* *} \mathrm{P}<0.01$ vs. control.

$\beta$-catenin notably alleviated meloxicam-induced suppression of MMP-2/9 upregulation and E-cadherin downregulation by treatment of PGE2 in HepG2 cells. A similar result was also found in SMMC-7721 cells (data not shown).

FH535, an inhibitor of $\beta$-catenin, suppresses PGE2-induced cell migration of HCC cells in vitro. To further investigate whether activation of $\beta$-catenin and PGE2 has a role in migration of HCC cells, HepG2 and SMMC-7721 cells were exposed to PGE2 with or without treatment with FH535, an inhibitor of $\beta$-catenin. As shown in Fig. 6A, treatment with PGE2 significantly increased the migration ability of HepG2 and SMMC-7721 cells. However, treatment of cells with FH535 markedly suppressed PGE2-enhanced migration of HCC cells. The results of western blotting showed that the level of MMP-2/9 was reduced and E-cadherin was enhanced 
by treatment with FH535 in HepG2 and SMMC-7721 cells (Fig. 6B and C). We also found similar effects in mRNA expression of MMP-2/9 and E-cadherin by RT-PCR assay (Fig. 6D).

\section{Discussion}

It was reported that $\mathrm{COX}-2$ is overexpressed in various cancers and is associated with cancer cell migration/invasion. The selective COX-2 inhibitor has been recognized as exerting antitumor effects through suppression of PGE2 production. COX-2 overexpression has been considered to be linked to cancer cell-derived PGE2 which promotes tumor cell proliferation, invasion, and migration and reduces apoptosis (37). Our previous studies also demonstrated that meloxicam produces its antitumor effects against hepatocellular carcinoma in COX-2-dependent and -independent pathways $(14,26)$. However, the exact anti-migration mechanism of meloxicam on the downstream pathway of PGE2 in HCC cells remains unknown. In this study, we found that $\mathrm{HCC}$ cell lines expressed different levels of COX-2 protein and that meloxicam exerted an anti-proliferation effect in HepG2 and SMMC-7721 cells via blocking the cell cycle in the $\mathrm{G} 1$ phase through regulating expression of $\mathrm{CDK}$ inhibitor proteins p21 and p27. Moreover, we observed that treatment with PGE2 significantly potentiated the migration potential whereas this effect was reversed after exposure to meloxicam, which suggested that meloxicam inhibition of the migration potential may be linked to the suppression of endogenous expression of COX-2 and production of PGE2 in HepG2 and SMMC-7721 cells. This concept is also supported by the evidence that transfection of HCC cells with COX-2 siRNA led to a marked reduction of cell migration in HepG2 and SMMC-7721 cells as compared to the migration of scramble siRNA-transfected HCC cells.

A large number of studies revealed that MMP-2/9 promote tumor metastasis and invasion by degrading extracellular matrix proteins $(38,39)$. In addition, E-cadherin has been reported as a suppressor of invasion and metastasis in many cancers (40). In the present study, we explored the protein and mRNA MMP-2/9 and E-cadherin expression following exposure to meloxicam with or without PGE2. Our results showed that expression of MMP-2 and MMP-9 was increased by PGE2 and reversed by meloxicam and the extent of E-cadherin was decreased following treatment with PGE2 whereas it was increased after exposed to meloxicam. We also observed similar effects in mRNA expression of MMP-2/9 and E-cadherin by RT-PCR assay. These data demonstrated that meloxicam has an inhibitory role in the migration and invasion of HCC cells through mediating the level of MMP-2/9 and E-cadherin.

Various studies reported the role of $\beta$-catenin in cancer invasion/migration through regulating cell-to-cell adhesion (41). The constitutively active $\beta$-catenin signaling pathway results in disturbed cell-to-cell adhesion and consequent upregulation of the migration potential of tumor cells. Moreover, some studies demonstrated that PGE2 could have pro-oncogenic actions including proliferation and metastasis by stimulating $\beta$-catenin-mediated transcription in carcinogenesis $(22,42)$. The data presented in the present study provide evidence that meloxicam exerts its anti-migration effects through downregulation of nuclear accumulation of $\beta$-catenin and inhibiting the phosphorylation of GSK-3 $\beta$. Knockdown of $\beta$-catenin by siRNA significantly reduced meloxicam-induced suppression of MMP-2/9 upregulation and E-cadherin downregulation by treatment of PGE2 in HepG2 and SMMC-7721 cells. To further explore the link between COX-2/PGE2 and $\beta$-catenin in HCC cell migration, we used FH535 (an inhibitor of $\beta$-catenin) to investigate whether PGE2-enhanced migration was dependent on the $\beta$-catenin signaling pathway. We observed that FH535 inhibited PGE2-enhanced migration of HCC cells.

In conclusion, the present study showed that meloxicam suppresses the migration of HCC cells by targeting PGE2regulated activation of the GSK-3 $\beta / \beta$-catenin signaling pathway. These findings suggest that meloxicam may be a potential therapeutic option for preventing HCC invasion/migration.

\section{Acknowledgements}

This research was supported from the National Natural Scientific Foundation of China (30972890 and 81172331), Shandong Provincial Science and Technology Development Planning, China (2015GGB14168) and Shandong Provincial Natural Science Foundation, China (ZR2015HL080). Thanks to Dr Edward C. Mignot, Shandong University, for linguistic advice.

\section{References}

1. Jemal A, Bray F, Center MM, Ferlay J, Ward E and Forman D: Global cancer statistics. CA Cancer J Clin 61: 69-90, 2011.

2. Forner A, Llovet JM and Bruix J: Hepatocellular carcinoma. Lancet 379: 1245-1255, 2012.

3. Bruix J, Gores GJ and Mazzaferro V: Hepatocellular carcinoma: Clinical frontiers and perspectives. Gut 63: 844-855, 2014.

4. Wang C, Jin G, Jin H, Wang N, Luo Q, Zhang Y, Gao D, Jiang K, $\mathrm{Gu} \mathrm{D}$, Shen Q, et al: Clusterin facilitates metastasis by EIF3I/ Akt/MMP13 signaling in hepatocellular carcinoma. Oncotarget 6: 2903-2916, 2015.

5. Yusup G, Akutsu Y, Mutallip M, Qin W, Hu X, KomatsuAkimoto A, Hoshino I, Hanari N, Mori M, Akanuma N, et al: A COX-2 inhibitor enhances the antitumor effects of chemotherapy and radiotherapy for esophageal squamous cell carcinoma. Int J Oncol 44: 1146-1152, 2014.

6. Zheng CY, Xiao W, Zhu MX, Pan XJ, Yang ZH and Zhou SY: Inhibition of cyclooxygenase-2 by tetramethylpyrazine and its effects on A549 cell invasion and metastasis. Int J Oncol 40: 2029-2037, 2012.

7. Qian M, Qian D, Jing H, Li Y, Ma C and Zhou Y: Combined cetuximab and celecoxib treatment exhibits a synergistic anticancer effect on human oral squamous cell carcinoma in vitro and in vivo. Oncol Rep 32: 1681-1688, 2014.

8. Chen Z, Liu M, Liu X, Huang S, Li L, Song B, Li H, Ren Q, Hu Z, Zhou Y, et al: COX-2 regulates E-cadherin expression through the NF- $\kappa$ B/Snail signaling pathway in gastric cancer. Int J Mol Med 32: 93-100, 2013.

9. Honjo S, Kase S, Osaki M, Ardyanto TD, Kaibara N and Ito $\mathrm{H}$ : COX-2 correlates with F-box protein, Skp2 expression and prognosis in human gastric carcinoma. Int J Oncol 26: 353-360, 2005.

10. Chen JY, Li CF, Kuo CC, Tsai KK, Hou MF and Hung WC: Cancer/stroma interplay via cyclooxygenase-2 and indoleamine 2,3-dioxygenase promotes breast cancer progression. Breast Cancer Res 16: 410, 2014.

11. Liu M, Li CM, Chen ZF, Ji R, Guo QH, Li Q, Zhang HL and Zhou YN: Celecoxib regulates apoptosis and autophagy via the PI3K/Akt signaling pathway in SGC-7901 gastric cancer cells. Int J Mol Med 33: 1451-1458, 2014.

12. Zhang H, Li Z and Wang K: Combining sorafenib with celecoxib synergistically inhibits tumor growth of non-small cell lung cancer cells in vitro and in vivo. Oncol Rep 31: 1954-1960, 2014. 
13. Xia Q, Hu Q, Wang H, Yang H, Gao F, Ren H, Chen D, Fu C, Zheng L, Zhen X, et al: Induction of COX-2-PGE2 synthesis by activation of the MAPK/ERK pathway contributes to neuronal death triggered by TDP-43-depleted microglia. Cell Death Dis 6: e1702, 2015.

14. Dong X, Li R, Xiu P, Dong X, Xu Z, Zhai B, Liu F, Jiang H, Sun X, Li J, et al: Meloxicam executes its antitumor effects against hepatocellular carcinoma in COX-2-dependent and -independent pathways. PLoS One 9: e92864, 2014.

15. Prasad R and Katiyar SK: Ultraviolet radiation-induced inflammation activates $\beta$-catenin signaling in mouse skin and skin tumors. Int J Oncol 44: 1199-1206, 2014.

16. Tu B, Ma TT, Peng XQ, Wang Q, Yang $H$ and Huang XL: Targeting of COX-2 expression by recombinant adenovirus shRNA attenuates the malignant biological behavior of breast cancer cells. Asian Pac J Cancer Prev 15: 8829-8836, 2014.

17. Jansen SR, Holman R, Hedemann I, Frankes E, Elzinga CR, Timens W, Gosens R, de Bont ES and Schmidt M: Prostaglandin E2 promotes MYCN non-amplified neuroblastoma cell survival via $\beta$-catenin stabilization. J Cell Mol Med 19: 210-226, 2015.

18. Shafie NH, Mohd Esa N, Ithnin H, Md Akim A, Saad N and Pandurangan AK: Preventive inositol hexaphosphate extracted from rice bran inhibits colorectal cancer through involvement of Wnt $/ \beta$-catenin and COX-2 pathways. Biomed Res Int 2013 : 681027,2013

19. Gavert $\mathrm{N}$ and Ben-Ze'ev A: beta-Catenin signaling in biological control and cancer. J Cell Biochem 102: 820-828, 2007.

20. Klaus A and Birchmeier W: Wnt signalling and its impact on development and cancer. Nat Rev Cancer 8: 387-398, 2008.

21. Jiang S, Zhu L, Tang H, Zhang M, Chen Z, Fei J, Han B and Zou GM: Ape1 regulates WNT/ $\beta$-catenin signaling through its redox functional domain in pancreatic cancer cells. Int $\mathrm{J}$ Oncol 47: 610-620, 2015

22. Du M, Shi F, Zhang H, Xia S, Zhang M, Ma J, Bai X, Zhang L, Wang Y, Cheng S, et al: Prostaglandin E2 promotes human cholangiocarcinoma cell proliferation, migration and invasion through the upregulation of $\beta$-catenin expression via EP3-4 receptor. Oncol Rep 34: 715-726, 2015.

23. Qiu X, Cheng JC, Chang HM and Leung PC: COX2 and PGE2 mediate EGF-induced E-cadherin-independent human ovarian cancer cell invasion. Endocr Relat Cancer 21: 533-543, 2014.

24. Zhang S, Da L, Yang X, Feng D, Yin R, Li M, Zhang Z, Jiang F and $\mathrm{Xu}$ L: Celecoxib potentially inhibits metastasis of lung cancer promoted by surgery in mice, via suppression of the PGE2-modulated $\beta$-catenin pathway. Toxicol Lett 225: 201-207, 2014.

25. Zhong J, Xiu P, Dong X, Wang F, Wei H, Wang X, Xu Z, Liu F, Li T, Wang Y, et al: Meloxicam combined with sorafenib synergistically inhibits tumor growth of human hepatocellular carcinoma cells via ER stress-related apoptosis. Oncol Rep 34 2142-2150, 2015

26. Zhong J, Dong X, Xiu P, Wang F, Liu J, Wei H, Xu Z, Liu F, Li T and Li J: Blocking autophagy enhances meloxicam lethality to hepatocellular carcinoma by promotion of endoplasmic reticulum stress. Cell Prolif 48: 691-704, 2015.

27. Wang F, Dong X, Xiu P, Zhong J, Wei H, Xu Z, Li T, Liu F, Sun X and Li J: T7 peptide inhibits angiogenesis via downregulation of angiopoietin-2 and autophagy. Oncol Rep 33: 675-684, 2015.
28. Wang L, Wang Z, Li J, Zhang W, Ren F and Yue W: NFATc1 activation promotes the invasion of U251 human glioblastoma multiforme cells through COX-2. Int J Mol Med 35: 1333-1340, 2015.

29. Park SY, Jin ML, Kim YH, Lee SJ and Park G: Sanguinarine inhibits invasiveness and the MMP-9 and COX-2 expression in TPA-induced breast cancer cells by inducing HO-1 expression. Oncol Rep 31: 497-504, 2014.

30. Liu X, Ji Q, Ye N, Sui H, Zhou L, Zhu H, Fan Z, Cai J and $\mathrm{Li}$ Q: Berberine inhibits invasion and metastasis of colorectal cancer cells via COX-2/PGE2 mediated JAK2/STAT3 signaling pathway. PLoS One 10: e0123478, 2015.

31. Lin X, Li HR, Lin XF, Yu ME, Tu XW, Hua ZD, Lin M, Xu NL, Han LL and Chen YS: Silencing of Livin inhibits tumorigenesis and metastasis via VEGF and MMPs pathway in lung cancer. Int J Oncol 47: 657-667, 2015.

32. Chou YC, Chang MY, Wang MJ, Yu FS, Liu HC, Harnod T, Hung $\mathrm{CH}$, Lee HT and Chung JG: PEITC inhibits human brain glioblastoma GBM 8401 cell migration and invasion through the inhibition of UPA, Rho A, and Ras with inhibition of MMP-2, -7 and -9 gene expression. Oncol Rep 34: 2489-2496, 2015.

33. Campbell $\mathrm{K}$ and Casanova J: A role for E-cadherin in ensuring cohesive migration of a heterogeneous population of nonepithelial cells. Nat Commun 6: 7998, 2015.

34. Fang Y, Liang X, Jiang W, Li J, Xu J and Cai X: Cyclin b1 suppresses colorectal cancer invasion and metastasis by regulating e-cadherin. PLoS One 10: e0126875, 2015.

35. Singh T and Katiyar SK: Honokiol inhibits non-small cell lung cancer cell migration by targeting $\mathrm{PGE}_{2}$-mediated activation of $\beta$-catenin signaling. PLoS One 8: e60749, 2013.

36. Yun SP, Ryu JM, Park JH, Kim MO, Lee JH and Han HJ: Prostaglandin $\mathrm{E}_{2}$ maintains mouse ESC undifferentiated state through regulation of connexin 31 , connexin 43 and connexin 45 expression: involvement of glycogen synthase kinase $3 \beta / \beta$-catenin. Biol Cell 104: 378-396, 2012.

37. Ho MY, Hung SW, Liang CM and Liang SM: Recombinant viral capsid protein VP1 suppresses lung cancer metastasis by inhibiting COX-2/PGE2 and MIG-7. Oncotarget 5: 3931-3943, 2014.

38. Lee CS, Cho HJ, Jeong YJ, Shin JM, Park KK, Park YY, Bae YS, Chung IK, Kim M, Kim CH, et al: Isothiocyanates inhibit the invasion and migration of C6 glioma cells by blocking FAK/JNK-mediated MMP-9 expression. Oncol Rep 34: 2901-2908, 2015

39. Chen F, Deng J, Liu X, Li W and Zheng J: HCRP-1 regulates cell migration and invasion via EGFR-ERK mediated up-regulation of MMP-2 with prognostic significance in human renal cell carcinoma. Sci Rep 5: 13470, 2015.

40. Peng X, Zhou Y, Tian H, Yang G, Li C, Geng Y, Wu S and Wu W: Sulforaphane inhibits invasion by phosphorylating ERK1/2 to regulate E-cadherin and CD44v6 in human prostate cancer DU145 cells. Oncol Rep 34: 1565-1572, 2015.

41. Tao H, Guo L, Chen L, et al.: MSX1 inhibits cell migration and invasion through regulating the Wnt/beta-catenin pathway in glioblastoma. Tumour Biol: Aug 15, 2015 (Epub ahead of print).

42. Yang C, Li C, Li M, Tong X, Hu X, Yang X, Yan X, He L and Wan C: CYP2S1 depletion enhances colorectal cell proliferation is associated with PGE2-mediated activation of $\beta$-catenin signaling. Exp Cell Res 331: 377-386, 2015. 